\title{
Sleep and Breathing Disturbance in Joubert Syndrome: A Case Report
}

\author{
Abdullah Saeed Al-Shamrani \\ Prince Sultan Military Medical City (PSMMC), Alfaisal University, Riyadh, Saudi Arabia
}

Email address:

dr.alshamrani99@gmail.com

\section{To cite this article:}

Abdullah Saeed Al-Shamrani. Sleep and Breathing Disturbance in Joubert Syndrome: A Case Report. American Journal of Pediatrics. Vol. 7, No. 2, 2021, pp. 79-84. doi: 10.11648/j.ajp.20210702.18

Received: May 6, 2021; Accepted: May 24, 2021; Published: May 31, 2021

\begin{abstract}
Joubert syndrome is a rare autosomal-recessive disease, and the description was coined by Dr. Marie Joubert in 1968 in a family presenting with breathing problems and certain central nervous system malformations in the form of hypotonia, cerebellar vermis hypoplasia, and developmental delay. There is a paucity of literature describing the clinical characteristics and types of sleep abnormalities in patients with Joubert's syndrome. We report the case of a young child observed to have breathing difficulties since the early neonatal period. He was initially diagnosed with seizure disorder, with little improvement with antiepileptic medications. The child showed significant psychomotor retardation. Our patient continued to have breathing disorders, mainly recurrent apnoea and nocturnal hypoxemia, which further worsened the child's development and mental status. A genetic study confirmed the diagnosis of Joubert's syndrome, and polysomnography further confirmed the severity of sleep disorder of breathing, which included predominantly central apnoea. Bilevel positive airway pressure (BiPAP) therapy was initiated and resulted in a marked improvement in the apnoea/hypopnoea index (AHI) and sleep quality, especially in the rapid eye movement (REM) stage. As the clinical manifestations proven to be atypical, it might be very challenging for health care providers to prove the diagnosis. We highlight some difficulties faced when encountering these patients, starting from diagnostic confirmation, therapeutic intervention to the prognosis and outcome of such condition.
\end{abstract}

Keywords: Joubert Syndrome, Apnoeas, Central Apnoea, Polysomnography (PSG), Hypoventilation, Sleep Disorders of Breathing (SDB)

\section{Introduction}

Joubert syndrome is an autosomal-recessive condition. The diagnosis of Joubert syndrome is often challenging and based on a unique combination of clinical features, MRI findings, and genetic confirmation. Joubert syndrome can have many manifestations [1-2], and the criteria commonly accepted currently for the diagnosis of pure Joubert's syndrome are as follows: [3-6]

\subsection{Presence of Molar Tooth Sign}

This can be confirmed by MRI and is due to the hypoplasia of the cerebellar vermis and long, thickened cerebellar peduncles in the axial plane. [2-4, 7, 8] MRI in the coronal and axial planes shows cerebellar vermis clefting.

\subsection{Hypotonia}

Hypotonia is present in all patients and is usually observed in early infancy. Gross motor function is markedly affected, with sitting achieved by 19 months and walking by 4 years; generally, hypotonia improves with time. [3-5, 9]

\subsection{Ataxia}

Almost universal in Joubert's syndrome, ataxia often manifests with a broad gait and marked difficulty running and walking upstairs due to cerebellar dysfunction. [3, 4, 9]

\subsection{Psychomotor Retardation (Mainly Intellectual)}

Psychomotor retardation is universal and affects a variety of domains, including general development, behaviour, language and intellectual ability. [3, 4, 5, 9] 


\subsection{Abnormal Breathing Pattern}

An irregular breathing pattern (tachypnoea/apnoea) is considered classic sign of JS and is reported in $44 \%-71 \%$ of patients. The breathing pattern of patients with JS has two components: a tachypnoeic phase, which is more common and is precipitated by emotional stress or handling, and an apnoeic phase, which is more serious as it could be prolonged for one minute. The breathing pattern of patients with Joubert syndrome is often not associated with cyanosis or bradycardia but was reported to occur more in the nonREM stage of sleep and in infancy and then to improve with age. $[3,4,9]$ Tachypnoea could persist until school age. [3, 4, $6,10,11]$

\subsection{Abnormal Eye Movements}

Typically, $70-100 \%$ of patients with JS have oculomotor apraxia; other manifestations vary and include nystagmus, ptosis, colobomas and night blindness. Retinal dysfunction is also common. [3, 4, 10]

\subsection{Typical Facial Features}

The following are commonly reported in patients with JS:

A triangular-shaped open mouth, with the tongue frequently resting on the lower lip; low-set and thick ears; high, rounded eyebrows; mild epicanthus; a broad nasal bridge; and anteverted nostrils. [2, 3, 10]

\subsection{Genetic Abnormalities}

Mutations in more than 34 genes are implicated in JS. Therefore, single-gene testing is NOT recommended because of the heterogenicity of the disease; instead, the Joubert panel is recommended. [12-18]

\subsection{Other Associated Anomalies}

1. Joubert syndrome with oculo-renal defects: These defects may include retinal dystrophy and coloboma, which lead to a loss of vision. The occurrence of polycystic kidney and end-stage renal disease requiring dialysis is reported to be as high as 50\%. [19-22]

2. Joubert syndrome with hepatic defects. [19]

3. Joubert syndrome with oro-facio-digital defects. $[19,20]$

It is really a great challenge to the paediatricians to diagnose the Joubert syndrome as many diseases could share the clinical presentations, this study presents a case report of a child with a psychomotor retardation and breathing disorder misdiagnosed as seizure disorder with highlight of diagnosing and treating problems which encountered in this patient.

\section{Case Illustration}

\subsection{History}

A 5-year-old boy with a known case of Joubert syndrome was referred to the sleep clinic for an evaluation of his breathing status. The history of this condition dated back to the neonatal period, when he had documented frequent apnoea and a seizure disorder, which was managed with antiepileptic medications with some clinical improvement. He was observed to have irregular breathing, which was faster during awake periods and included frequent cyanotic spells during sleep periods; for this, he was treated with caffeine. At the end of the first year, snoring, along with apnoea and restlessness, was witnessed during sleep. The first sleep study in our centre was done as a diagnostic study at the age of one-year due irregular breathing. The study was suboptimal, as the child has recent URTI three days prior to the study, with a total study time of $5.5 \mathrm{~h}$. Breathing during the REM stage was very low, with an alarming AHI of $9.1 / \mathrm{h}$, and for this he was treated with nasal inhaled steroid and saline and referred to the ENT department. In the ENT department, the family was reassured that his condition was not an indication for adenotonsillectomy. He was taken to the UK, where he was genetically confirmed to have Joubert syndrome (CC2D2A, C. 3364C $>$ T; p. P1122S). Further, he was diagnosed with sleep disorder of breathing and received a BiPAP machine. He was lost to follow-up, and even lost the connection to the BiPAP machine. At the age of 5 years, the patient, with clear psychomotor retardation, arrived at our clinic for further evaluation of his breathing problem.

\subsection{On Examination}

The patient has obvious intellectual abnormalities, along with the characteristic facial features of Joubert's syndrome, including a broad forehead, triangular face, low-set ears, ptosis and hypertelorism. His weight was just below the third centile. His breathing was regular (25/minute), with $98 \%$ saturation in room air, and he showed clear chest vesicular breathing with no added adventitious sounds. He had mild hypotonia, mild ataxic gait and wore eyeglasses. No further abnormalities were observed.

\subsection{Investigations}

We scheduled his second sleep study on 2 Jan 2021 and confirmed the following: decreased sleep efficiency, $72 \%$; marked reduced REM, 12\%; and mild nocturnal hypoxemia with severe SDB, where the AHI was $17.3 / \mathrm{h}$, with mainly central apnoea. He further underwent a titration study on 7 March 2021. The findings were consistent with severe SDB with, an AHI of $12.4 / \mathrm{h}$, with mainly central apnoea, which is expected as part of the underlying disease. The result was similar to the January study. Fortunately, all respiratory events were eliminated with the BiPAP ST mode. Central apnoea was defined as a reduction in airflow of $>80 \%$, associated with no evidence of abdominal or chest wall motion, lasting 20 seconds in duration or shorter if associated with a $>3 \%$ drop in oxygen saturation.

\subsection{The Recommendations}

To start, for the BiPAP ST mode, a pressure of $15 / 8 \mathrm{~cm}$ $\mathrm{H}_{2} \mathrm{O}$ with a backup rate of $12 / \mathrm{m}$ was recommended during 
sleep. From the study, sleep onset was significantly delayed, up to 63 minutes, and sleep efficiency was low, but the REM stage was much longer than in previous study $(26 \%)$, which could be explained by the noninvasive BiPAP intervention. All sleep stages were seen, with no significant nocturnal hypoxemia. $\mathrm{ETCO}_{2}$ was inaccurate. $\mathrm{TCCO}_{2}$ was normal, and no evidence of nocturnal hypoventilation was noted. He was referred to home care, but unfortunately, the mask was not available. We hope to start the regular follow-up of the patient in the clinic with a multidisciplinary team soon.

Sleep study

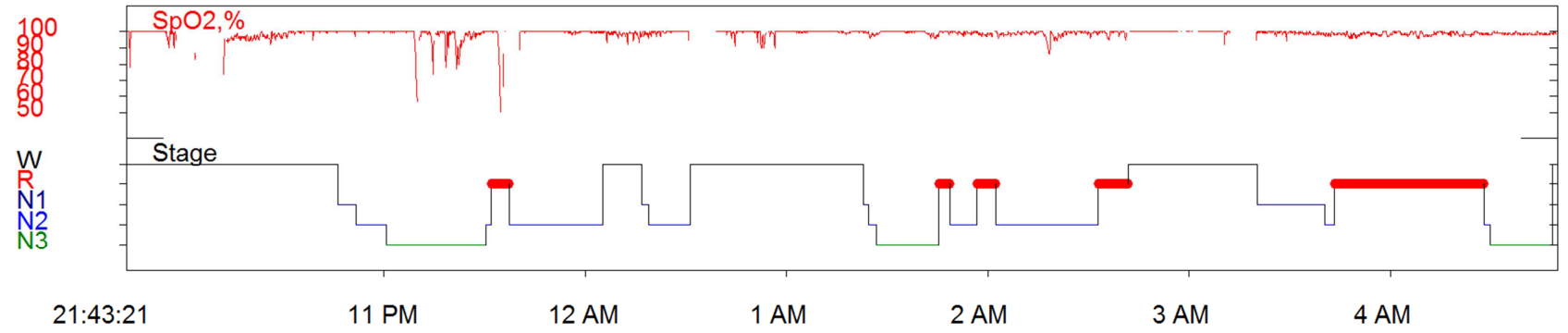

Figure 1. Hypnogram showing a cluster of desaturation in the first third of the night and marked improvement in sleep quality and a reduction of the event in the last third of the night after connection to the BiPAP.

Table 1. The presence of central apnoea and a significant $\mathrm{AHI}$ with marked improvement at end of the study, with an IPAP of 15 and an EPAP of $8 \mathrm{~cm}$ of $\mathrm{H}_{2} \mathrm{O}$ with a normal saturation.

\begin{tabular}{|c|c|c|c|c|c|c|c|c|c|c|c|c|c|c|c|}
\hline \multirow{2}{*}{ IPAP } & \multirow{2}{*}{$\begin{array}{l}\text { EP } \\
\text { AP }\end{array}$} & \multirow{2}{*}{$\begin{array}{l}\text { TIB } \\
(\mathrm{min})\end{array}$} & \multirow{2}{*}{$\begin{array}{l}\text { Sleep } \\
(\mathrm{min})\end{array}$} & \multirow{2}{*}{$\begin{array}{l}\text { REM } \\
\text { (min) }\end{array}$} & \multicolumn{4}{|c|}{ Apneas } & \multicolumn{2}{|c|}{ Hypopneas } & \multicolumn{2}{|c|}{ RERAs } & \multirow{2}{*}{ AHI } & \multirow{2}{*}{ RDI } & \multirow{2}{*}{$\begin{array}{l}\text { Minimum } \\
\text { SpO2 }\end{array}$} \\
\hline & & & & & $\mathbf{C A}$ & OA & MA & Index & $\#$ & Index & $\#$ & Index & & & \\
\hline 4 & 4 & 73.2 & 17.3 & 0.0 & 5 & 0 & 0 & 17.3 & 0 & 0.0 & 0 & 0.0 & 17.3 & 17.3 & 89 \\
\hline 5 & 5 & 3.0 & 3.0 & 0.0 & 0 & 0 & 0 & 0.0 & 0 & 0.0 & 0 & 0.0 & 0.0 & 0.0 & 97 \\
\hline 6 & 6 & 6.5 & 6.5 & 0.0 & 0 & 0 & 0 & 0.0 & 0 & 0.0 & 0 & 0.0 & 0.0 & 0.0 & 99 \\
\hline 7 & 7 & 5.1 & 5.1 & 0.0 & 1 & 0 & 0 & 11.8 & 0 & 0.0 & 0 & 0.0 & 11.8 & 11.8 & 99 \\
\hline 8 & 4 & 8.0 & 8.0 & 0.0 & 3 & 0 & 0 & 22.5 & 0 & 0.0 & 0 & 0.0 & 22.5 & 22.5 & 97 \\
\hline 8 & 8 & 5.5 & 5.5 & 1.6 & 3 & 0 & 0 & 32.7 & 0 & 0.0 & 0 & 0.0 & 32.7 & 32.7 & 96 \\
\hline 9 & 5 & 6.1 & 6.1 & 0.0 & 1 & 0 & 0 & 9.8 & 0 & 0.0 & 0 & 0.0 & 9.8 & 9.8 & 89 \\
\hline 9 & 9 & 6.5 & 6.5 & 1.6 & 2 & 0 & 0 & 18.5 & 0 & 0.0 & 0 & 0.0 & 18.5 & 18.5 & 97 \\
\hline 10 & 6 & 3.3 & 3.3 & 0.0 & 0 & 0 & 0 & 0.0 & 1 & 18.2 & 0 & 0.0 & 18.2 & 18.2 & 85 \\
\hline 11 & 6 & 6.4 & 6.4 & 0.0 & 0 & 0 & 0 & 0.0 & 1 & 9.4 & 0 & 0.0 & 9.4 & 9.4 & 96 \\
\hline 11 & 11 & 8.9 & 8.9 & 5.5 & 2 & 0 & 0 & 13.5 & 0 & 0.0 & 0 & 0.0 & 13.5 & 13.5 & 96 \\
\hline 12 & 6 & 4.0 & 4.0 & 0.0 & 2 & 0 & 0 & 30.0 & 0 & 0.0 & 0 & 0.0 & 30.0 & 30.0 & 96 \\
\hline 13 & 7 & 2.4 & 2.4 & 1.9 & 1 & 0 & 0 & 25.0 & 0 & 0.0 & 0 & 0.0 & 25.0 & 25.0 & 98 \\
\hline 14 & 8 & 48.1 & 29.3 & 6.5 & 9 & 0 & 1 & 20.5 & 0 & 0.0 & 0 & 0.0 & 20.5 & 20.5 & 92 \\
\hline 15 & 8 & 63.7 & 63.7 & 43.2 & 0 & 0 & 0 & 0.0 & 0 & 0.0 & 0 & 0.0 & 0.0 & 0.0 & 96 \\
\hline
\end{tabular}

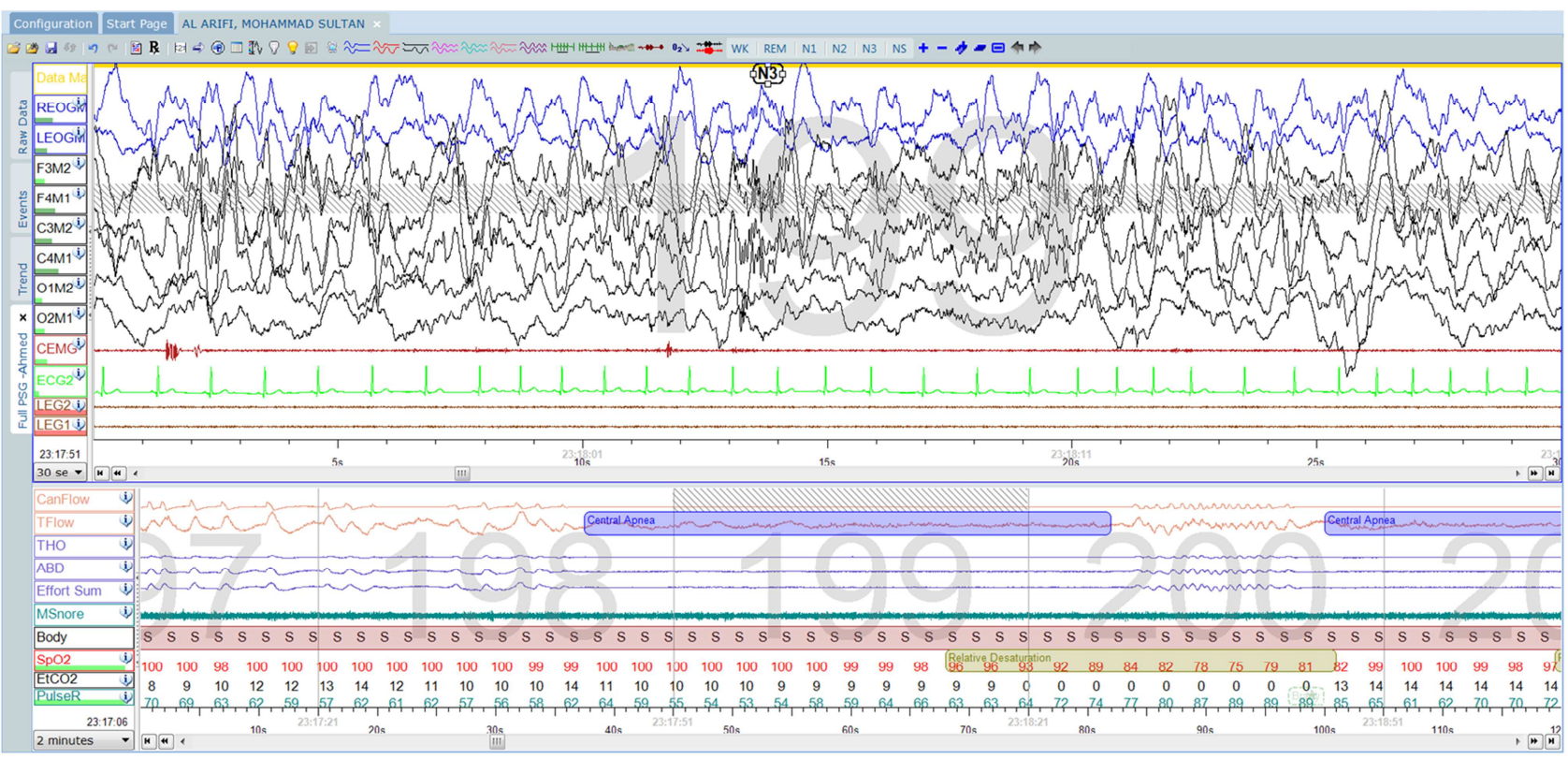

Figure 2. Polysomnography 2-minute epoch: N3 with central apnoea with significant desaturation. 


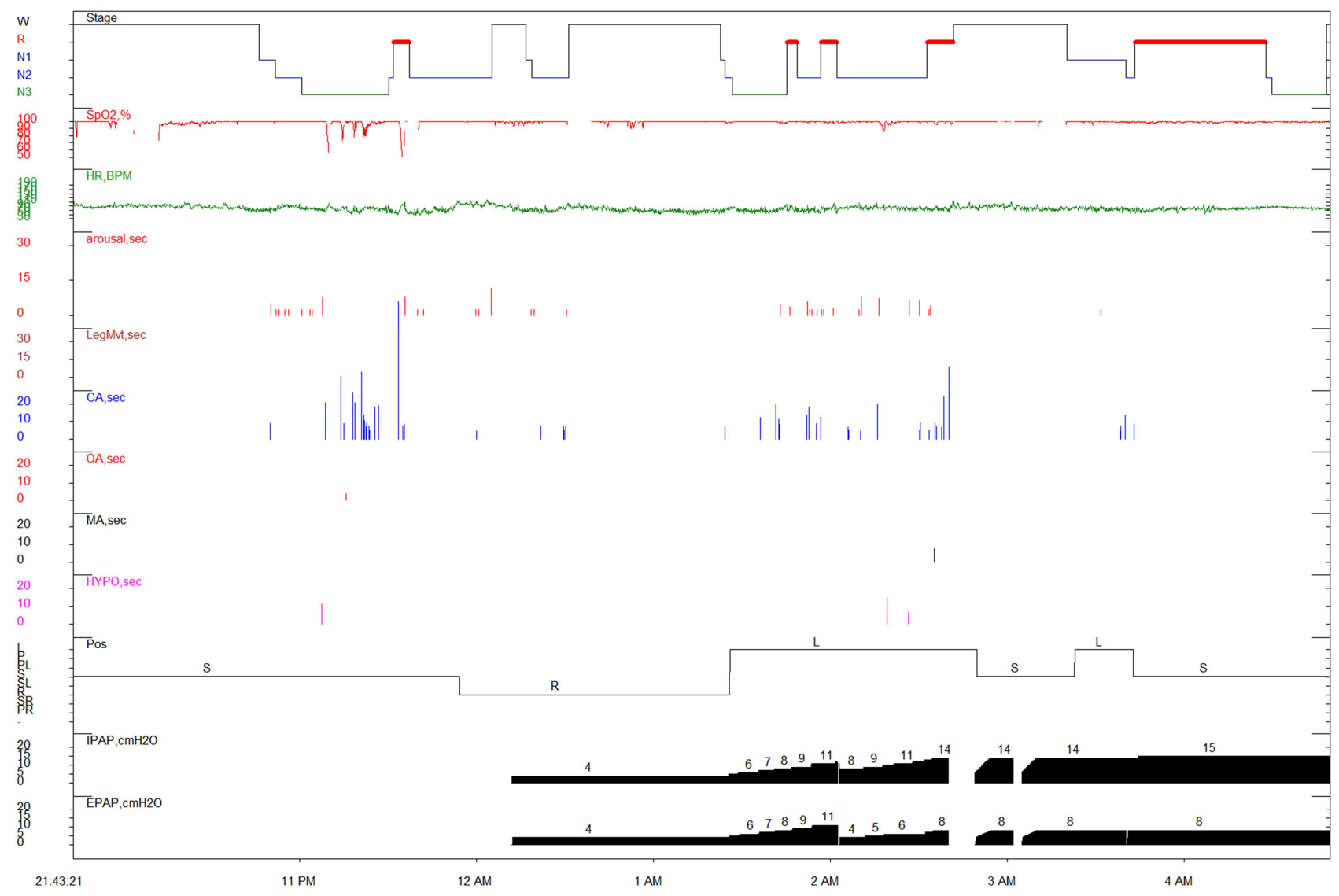

Figure 3. Hypnogram showing the results of the titration study and confirming the resolution of the central apnoea with BiPAP and a marked improvement in sleep quality in the last third of the night.

\section{Discussion}

Literature reviewing Joubert syndrome in the paediatric population is limited and quite recent. Our case met the clinical criteria for Joubert syndrome, and this diagnosis was confirmed by a genetic study.

Hypotonia and ataxia are common in paediatric patients, and a wide range of diseases with acquired or congenital causes can result in these conditions; thus, additional features are needed to diagnose Joubert's syndrome. [3-5] Our patient had hypotonia and some cerebellar dysfunction, and he started to suffer from frequent cyanotic spells in the neonatal period. He was initially diagnosed with seizure disorder, with little improvement on antiepileptic medication, and the neonatologist started respiratory stimulation with caffeine, but with modest benefit. An extensive workup was performed to rule out other potential causes, including brain imaging to rule out structural brain diseases, barium imaging to rule out gastrointestinal causes such as gastroesophageal reflux disease (GERD), and metabolic screening to rule out metabolic causes. The MRI showed the typical molar sign of Joubert's syndrome $[7,8]$, the metabolic screening was negative, and barium imaging was normal, although our patient received a proton pump inhibitor without much improvement. The parents observed the patient snore, at least three nights a week, with occasional witnessed apnoea and restlessness during sleep. While awake, the child was hyperactive compared to the sibling. The patient was seen by an otolaryngologist, who reassured the parents that the patient's condition was not an indication for adenotonsillectomy. A second opinion was obtained in the UK, and the genetic analysis confirmed the diagnosis of Joubert's syndrome. The mutation was similar to that reported by Nicholas T. Gorden et al in 2008 and occurred in the CC2D2A gene (MIM 612013). [17] Reviewing the genes involved in Joubert syndrome can be confusing, as mutations in more than 34 genes are implicated in the disease. Therefore, single-gene testing is rarely useful and typically NOT recommended because of the heterogeneity of the disease; instead, the Joubert panel is recommended. [12-18] Common reported mutations are found in the CEP290 [MIM 610142], AHI1 [MIM 608894], ARL13B [MIM 608922], NPHP1 [MIM 607100], RPGRIP1L [MIM 610937], and TMEM67 [MIM 609884] genes, all of which have been implicated in the function of the primary cilium and JS. [17] Nevertheless, there is a paucity of literature on sleep-related problems and breathing abnormalities in patients with Joubert syndrome.

One of the best studies was a retrospective review of the cases of 11 patients based on clinical and polysomnography; the main reasons for referral were witnessed apnoea $(73 \%)$, snoring (64\%), and desaturation (36\%) [23, 24]. Recently, J. Cruz et al described the breathing pattern on PSG as periods of hyperventilation followed by a short period of central apnoea in both sleep and awake states; these findings were 
similar to those in our patient observed by the patient's mother. The severity of the AHI was slightly less than that reported in literature $(13 \%$ vs $23 \%)$. Further, our patient showed predominantly central apnoea, while in the literature, only 45\% central apnoea was observed. [25] Another alarming figure is that obstructive apnoea was reported to be as high as $82 \%$, while it was very low in our patient. [25] It is not known whether prolonged apnoea may cause death, although this has been reported in earlier literature. [9]

No significant hypoventilation or PLMD was observed in our patient. The outcome of JS is still variable, and the cause of death is often either respiratory failure or renal failure. [3, 21] These anomalies could be progressive and need close monitoring. Physical, occupational, and speech therapy may be helpful for some individuals.

\section{Conclusion}

This study emphasizes that individuals with Joubert syndrome suffer from significant central sleep apnoea and nocturnal hypoxemia, which could further adversely affect their mental status. Our case is similar to the few reported cases highlighting the severe sleep disturbance in individuals with Joubert syndrome. We recommend routine diagnostic studies starting from infancy. Patients with central apnoea often need titration with either CPAP or BiPAP. This report highlights difficulties in patients with late diagnoses and in starting sleep interventions. We are confident that understanding the sleep and breathing abnormalities in individuals with JS will improve their outcome and quality of life.

\section{Abbreviations}

PSG: Polysomnography

SDB: Sleep disorders of breathing

GERD: Gastroesophageal reflux disease

AHI: Apnea hypopnea index

REM: Rapid eye movement

$\mathrm{ETCO}_{2}$ : End tidal carbon dioxide

$\mathrm{TCCO}_{2}$ : transcutaneous carbo dioxide

BiPAP: Bilevel positive airway pressure

\section{Conflict of Interest}

The author does not have any possible conflicts of interest.

\section{Consent for Publication}

Written informed consent was obtained from all the participants.

\section{Ethics Approval}

Signed informed consent for participation and the publication of medical details was obtained from the parents of this child. The confidentiality of the patient's data was ensured at all times.

\section{References}

[1] Joubert M, Eisenring JJ, Robb JP, Andermann F. Familial agenesis of the cerebellar vermis. A syndrome of episodic hyperpnea, abnormal eye movements, ataxia, and retardation. Neurology 1969; 19: 813-25.

[2] Maria BL, Hoang KB, Tusa RJ, et al. "Joubert syndrome" revisited: key ocular motor signs with magnetic resonance imaging correlation. J Child Neurol 1997; 12: 423-30.

[3] Maria BL, Boltshauser E, Palmer SC, Tran TX. Clinical features and revised diagnostic criteria in Joubert syndrome. $J$ Child Neurol 1999; 14: 583-90.

[4] Saraiva JM, Baraitser M. Joubert syndrome: a review. Am J Med Genet 1992; 43: 726-31. 13.

[5] Merritt L. Recognition of the clinical signs and symptoms of Joubert syndrome. Adv Neonatal Care. 2003; 3: 178-86.

[6] Elhassanien, AhmedFarag; Alghaiaty, HeshamAbdel-Aziz (2013). Joubert syndrome: Clinical and radiological characteristics of nine patients. Annals of Indian Academy of Neurology, 16 (2), 239.

[7] Gleeson JG, Keeler LC, Parisi MA, et al. Molar tooth sign of the midbrain-hindbrain junction: occurrence in multiple distinct syndromes. Am J Med Genet A 2004; 1125-34.

[8] Valente EM, Salpietro DC, Brancati F, et al. Description, nomenclature, and mapping of a novel cerebello-renal syndrome with the molar tooth malformation. Am J Hum Genet 2003; 73: 663-70.

[9] Pellegrino JE, Lensch MW, Muenki M, Chance PF: Clinical and molecular analysis in Joubert syndrome. Am J Med Genet 1997; 72: 59-62.

[10] Steinlin, M.; Schmid, M.; Landau, K.; Boltshauser, E. (1997). Follow-Up in Children with Joubert Syndrome. Neuropediatrics, 28 (4), 204-211.

[11] Boltshauser E, Lange B, Dumermuth G: Differential diagnosis of syndromes with abnormal respiration (tachypnea-apnea). Brain Dev 1987; 9: 462-465.

[12] Brancati F, Barrano G, Silhavy JL, et al. CEP290 mutations are frequently identified in the oculo-renal form of Joubert syndrome-related disorders. Am J Hum Genet 2007; 81: 10413.

[13] Bachmann-Gagescu J C Dempsey, I G Phelps, BJO'Roak, et $a l$ : Joubert syndrome: a model for untangling recessive disorders with extreme genetic heterogeneity. 2015; J Med Genet; 52: 514-522.

[14] Vilboux et al (2017) identified pathogenic variants in 81 (94\%) of 86 families tested (100 individuals total) using a combination of 27- gene multigene panel and exome sequencing.

[15] Jo Melissa Parisi, and Ian Glass. Joubert Syndrome and Related Disorders (JSRD). [Updated 2017 Jun 29]. In: Adam MP, Ardinger HH, Pagon RA, et al., editors. Gene Reviews [Internet]. Seattle (WA): University of Washington, Seattle; 1993-2021. Bookshelf URL: https://www.ncbi.nlm.nih.gov/books/. 
[16] Keeler LC, Marsh SE, Leeflang EP, et al. Linkage analysis in families with Joubert syndrome plus oculo-renal involvement identifies the CORS2 locus on chromosome 11 p12-q13.3. Am J Hum Genet 2003; 73: 656-62.

[17] Gorden NT, Arts HH, Parisi MA, et al. CC2D2A is mutated in Joubert syndrome and interacts with the ciliopathy-associated basal body protein CEP290. Am J Hum Genet 2008; 83: 55971.

[18] Cantagrel V, Silhavy JL, Bielas SL, et al. Mutations in the cilia gene ARL13B lead to the classical form of Joubert syndrome. Am J Hum Genet 2008; 83: 170-9.

[19] Parisi MA, Doherty D, Chance PF, Glass IA. Joubert syndrome (and related disorders) (OMIM 213300). Eur J Hum Genet 2007; 15: 511-21.

[20] Justyna Paprocka, Ewa Jamroz, Joubert syndrome and related disorders. Neurologia Neurochirurgia Polska 2012; 46, 4: 379-383.
[21] Boltshauser E, Forster I, Deonna T, Willi U: Joubert syndrome: Are kidneys involved? Neuropediatrics 1995; 26: 320-321.

[22] Francesco Brancati; Bruno Dallapiccola; Enza Maria Valente: Joubert Syndrome and related disorders. Orphanet J Rare Dis. 2010; 5 (1), 20-9.

[23] Rana, Mandeep; Mohanty, Mugdha (2018). The spectrum of sleep disordered breathing in Joubert Syndrome. Sleep Medicine, 52 (2018), 177-179.

[24] Pascoe JE, Sarber K, Dye T, Simakajornboon: sleep disordered breathing in pediatric patient with Joubert's syndrome: sleep 2018; (41): A289.

[25] J. Cruz, D. G. Ingram, B. Al-Shawwa: sleep and Breathing Abnormalities in a Child with Joubert Syndrome. Am J Respir Crit Care Med 2020; 201: 7620. 